\title{
New Obligations of Health Insurance Review and Assessment Service: Taking Full-fledged Action Against the COVID-19 Pandemic
}

\author{
Seung Mi Yoo ${ }^{1 *}$, Seol Hee Chung ${ }^{1 *}$, Won Mo Jang ${ }^{2}$, Kyoung Chang Kim ${ }^{1}$, Jin Yong Lee ${ }^{3,4,5}$, Sun Min Kim ${ }^{6}$ \\ ${ }^{\prime}$ International Cooperation Department, Health Insurance Review and Assessment Service, Wonju, Korea; ${ }^{2}$ Department of Public Health and \\ Community Medicine, Seoul Metropolitan Government-Seoul National University Boramae Medical Center, Seoul, Korea; ${ }^{3} \mathrm{HIRA}$ Research Institute, \\ Health Insurance Review and Assessment Service, Wonju, Korea; ${ }^{4}$ Public Healthcare Center, Seoul National University Hospital, Seoul, Korea; \\ ${ }^{5}$ Department of Health Policy Management, Seoul National University College of Medicine, Seoul, Korea; ${ }^{6}$ Health Insurance Review and Assessment \\ Service, Wonju, Korea
}

In 2020, the coronavirus disease 2019 (COVID-19) pandemic has caused unprecedented disruptions to global health systems. The Korea has taken full-fledged actions against this novel infectious disease, swiftly implementing a testing-tracing-treatment strategy. New obligations have therefore been given to the Health Insurance Review and Assessment Service (HIRA) to devote the utmost effort towards tackling this global health crisis. Thanks to the universal national health insurance and state-of-the-art information communications technology (ICT) of the Korea, HIRA has conducted far-reaching countermeasures to detect and treat cases early, prevent the spread of COVID-19, respond quickly to surging demand for the healthcare services, and translate evidence into policy. Three main factors have enabled HIRA to undertake pandemic control preemptively and systematically: nationwide data aggregated from all healthcare providers and patients, pre-existing ICT network systems, and real-time data exchanges. HIRA has maximized the use of data and pre-existing network systems to conduct rapid and responsive measures in a centralized way, both of which have been the most critical tactics and strategies used by the Korean healthcare system. In the face of new obligations, our promise is to strive for a more responsive and resilient health system during this prolonged crisis.

Key words: SARS-CoV-2, Pandemics, Emerging infectious disease, Universal health coverage, Information technology, COVID-19

In 2020, the coronavirus disease 2019 (COVID-19) pandemic has caused unprecedented disruptions to global health systems, revealing systemic weaknesses and even causing the

Received: December 3, 2020 Accepted: January 7, 2021

Corresponding author: Sun Min Kim

Health Insurance Review and Assessment Service, 60 Hyeoksin-ro, Wonju 26465, Korea

E-mail: kimsunmin.sarah@gmail.com

* Yoo and Chung contributed equally to this work as joint first authors.

This is an Open Access article distributed under the terms of the Creative Commons Attribution Non-Commercial License (https://creativecommons.org/licenses/by$\mathrm{nc} / 4.0 /$ ) which permits unrestricted non-commercial use, distribution, and reproduction in any medium, provided the original work is properly cited. collapse of some health systems. This crisis has accelerated the pre-existing momentum to strengthen health systems in order to ensure robust and resilient health systems across the world.

The Korea has taken full-fledged actions against the COVID-19 crisis, swiftly implementing a testing-tracing-treatment (3T) strategy from early on in the pandemic [1-3]. In this tumultuous time, Korea's National Health Insurance (NHI) has emerged as a key factor in tackling COVID-19 with the 3T strategy from January 2020 to July 2020. Korea achieved universal health coverage for the entire population in 1989, and integrated into a single-payer system in 2000. The Heath In- 
surance Review and Assessment Service (HIRA) was established to determine health benefit packages and conduct expert claim reviews and quality assessment of the $\mathrm{NHI}[4,5]$.

Other distinctive characteristics of the NHI differentiate the Korean strategies from those adopted in other countries. Under the NHI Act, all healthcare providers in Korea are obligated to enroll in the single-payer $\mathrm{NHI}$ and submit claims data to HIRA for reimbursement [4-6]. In addition, the NHI system adopted advanced information and communications technology (ICT) in its early stages to ensure transparency and accountability $[5,6]$. Therefore, HIRA is able to collect and link health data across the country, utilize it for further applications, and promote data-driven policy responses.

As such, new obligations have been given to HIRA to manage this global health crisis. HIRA has utilized the following three main factors to undertake pandemic control pre-emptively and systematically: nationwide data aggregated from all healthcare providers and patients, the pre-existing ICT network, and the real-time data exchanges. By promptly setting up the emergency response framework, HIRA took its first step against COVID-19 on January 10, 2020, well before Korea's first case. This proactive response continued throughout the pandemic, allowing HIRA to devote the utmost effort towards managing the crisis and ensuring health system resilience (Figure 1).

First, no individuals in Korea face cost barriers when receiving COVID-19 testing and treatment, making healthcare extremely accessible and affordable. Even in countries with universal health coverage, an emergency response takes time to make the decisions to cover testing and treatment for a novel infectious disease. Upon the emergency use authorization of the Korea Ministry of Food and Drug Safety, HIRA dramatically expedited the $\mathrm{NHI}$ coverage process in response to the urgent need for COVID-19 testing within 3 days and medications within 1 day. Accordingly, the Korean NHI covers all of the costs pertaining to COVID-19 testing and treatment. The central and local governments cover any remaining out-of-pocket expenses. Patients are quickly able to receive testing and treatment, which is the most important factor in containing the virus.

Second, HIRA detects COVID-19 high-risk groups within healthcare settings with its real-time data exchange feature. The primary system is drug utilization review (DUR), which was originally implemented to transmit drug safety information at the time of prescribing and dispensing drugs. During the Middle East respiratory syndrome crisis in 2015, HIRA added an additional function, focusing the exceptional real-time data exchange feature of the DUR on notifying susceptible patients with a travel history to the Middle East region [6]. Later in 2018, the Korea Centers for Disease Control and Prevention (KCDC) and HIRA jointly developed a supplementary system called the International Traveler Information System (ITS) to provide this information at the reception and consultation stages before prescribing drugs. After the official notification of cases of pneumonia of unknown source detected in Wuhan, China on December 31, 2019, the Korean government was immediately informed to take actions [2]. Thus, since January 10, 2020, HIRA has provided access to data on high-risk patients, including overseas travel history and close contact history with positive cases, along with DUR and ITS. Through these systems, frontline healthcare providers can easily detect highrisk patients vulnerable to COVID-19, protecting them from potential infection and thereby eventually preventing the collapse of the healthcare system. HIRA also provides patients' history of medical visits to the KCDC for contact tracing to prevent community spread [7].

Third, HIRA has contributed to stabilizing the nationwide distribution of face masks, flexibly increasing the functionality of the system to align with needs during the emergency. Following the government decision to take full control of face mask distribution in early March, HIRA swiftly set up the National Face Mask Inventory System [8]. Although face masks are not covered by the NHI scheme, HIRA has been able to take full advantage of the pre-existing network systems with nationwide pharmacies to stabilize mask distribution to the public. This system allowed providers to establish and check the weekly purchase histories of each person in order to prevent hoarding and overcrowded pharmacies.

Fourth, HIRA implemented the National Health Resources Dashboard system for COVID-19 in mid-March, which was triggered by the surge in healthcare services. This was possible because all healthcare providers in Korea are required report their status of health resources such as workforce, facility, and equipment to HIRA for claims and reimbursement purposes. This dashboard system has provided daily snapshots of available resources, including negative-pressure isolation rooms, extracorporeal membrane oxygenation machines, and ventilators in order to easily identify hospitals with available resources and quickly relocate patients in a critical condition to optimize the COVID-19 patient flow. 

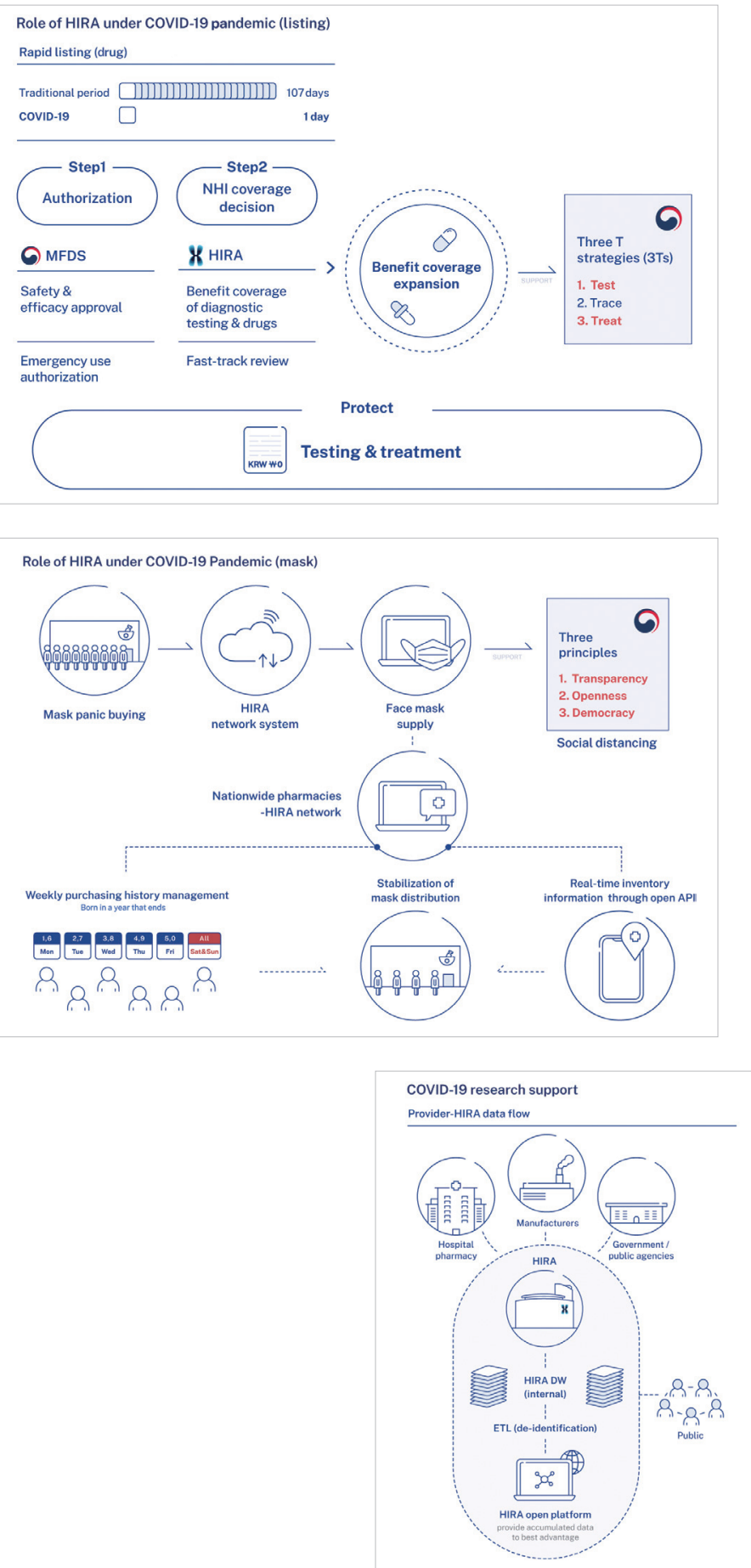

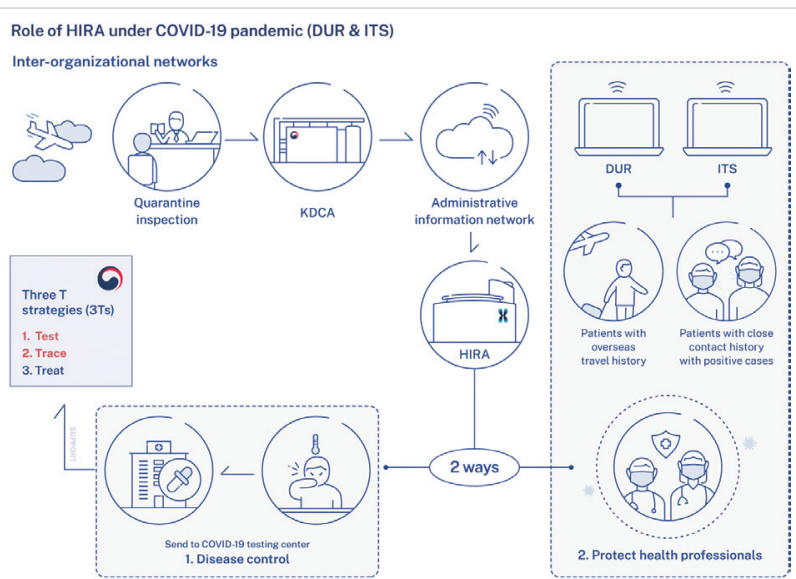

Role of HIRA under COVID-19 pandemic (health resources allocation)
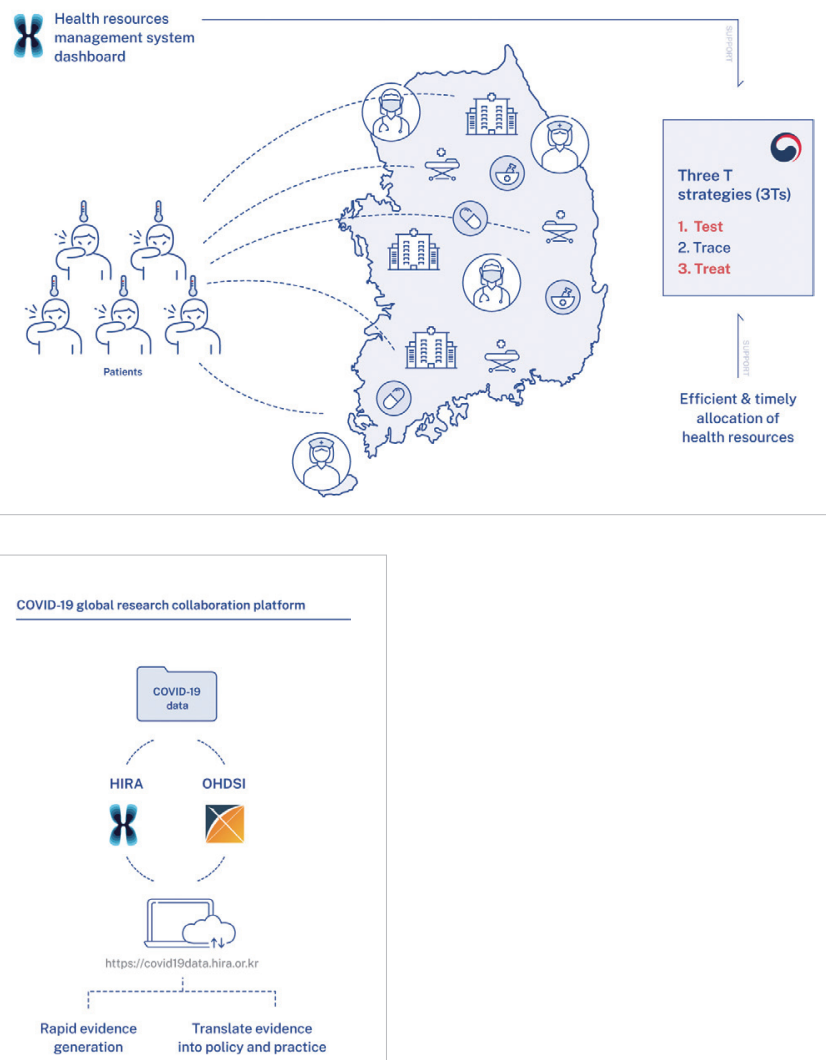

Figure 1. 5 Strategies of HIRA in response to coronavirus disease 2019 (COVID-19). NHI, National Health Insurance; MFDS, Ministry of Food and Drug Safety; HIRA, Health Insurance and Assessment Service; KDCA, Korea Disease Control and Prevention Agency; DUR, drug utilization review; ITS, International Traveler Information System; DW, data warehouse; ETL, extract, transformation, load; OHDSI, Observational Health Data Sciences and Informatics.

Fifth, HIRA has implemented the national COVID-19 patient data repository to collect data, make decisions in a centralized way, and share data to generate evidence to tackle this global health crisis. This repository was developed to monitor patient status in real-time and to adjust national COVID-19 response measures. As all healthcare providers in Korea submit claims, 
HIRA is able to collect and process real-world datasets from administrative claims data to build the COVID-19 Global Research Collaboration Platform. HIRA has shared the world's first de-identified nationwide COVID-19 patient dataset with researchers from all over the world for use in over 390 research projects to produce evidence for data-driven decision making.

In sum, these five strategies implemented by HIRA clearly indicate that the universal NHI system and robust ICT system have played a pivotal role in Korea's proactive response to the COVID-19 pandemic. Further, HIRA has maximized the use of data and pre-existing network systems to conduct rapid and responsive measures in a centralized way.

The COVID-19 pandemic is still raging throughout the world. It remains unclear how and when this crisis will end. In the face of new obligations, our promise is to strive for a more responsive and resilient health system during this prolonged crisis. Considering this, we are committed to a series of strategies to continue towards defeating the COVID-19 pandemic. First, we will fast-track the coverage process for COVID-19 therapeutics upon development to treat patients and protect healthcare professionals. Second, HIRA will devote itself to building a robust and real-time emergency response system for national health resources, reducing the delay between crisis and resource allocation. It is crucial to establish an always-on emergency response system within HIRA as a reservoir. Third, to respond to future emergencies, we will constantly expand data distribution channels, improve interconnectivity, and make data easily accessible to health professionals, researchers, and health authorities around the world. Overall, this will facilitate the generation of scientific evidence and clinical data for unknown diseases and inform policy and practices to tackle this crisis together.

\section{Ethics Statement}

The Institutional Review Board of Seoul Metropolitan Government, Seoul National University, Boramae Medical Center (No. 20200403/07-2020-12/043) approved this study. Th requirement for informed consent was waived, as this study was a perspective on strategies performed by HIRA.

\section{CONFLICT OF INTEREST}

The authors have no conflicts of interest associated with the material presented in this paper.

\section{FUNDING}

None.

\section{ACKNOWLEDGEMENTS}

None.

\section{AUTHOR CONTRIBUTIONS}

Conceptualization: SMY, SHC, SMK, JYL. Formal analysis: SMY, SHC, WMJ. Funding acquisition: None. Visualization: KCK. Writing - original draft: SMY, SHC, SMK, JYL. Writing - review \& editing: SMY, SHC, WMJ, KCK, SMK, JYL.

\section{ORCID}

Seung Mi Yoo https://orcid.org/0000-0003-4080-5543

Seol Hee Chung https://orcid.org/0000-0002-4879-8942

Won Mo Jang https://orcid.org/0000-0003-3277-7706

Kyoung Chang Kim https://orcid.org/0000-0002-1985-276X

Jin Yong Lee https://orcid.org/0000-0002-7752-2697

Sun Min Kim https://orcid.org/0000-0002-5056-0570

\section{REFERENCES}

1. Ahn MJ. Combating COVID-19: lessons from South Korea; 2020 [cited 2020 Oct 20]. Available from: https://www.brookings. edu/blog/techtank/2020/04/13/combating-covid-19-lessonsfrom-south-korea/.

2. Lee $D$, Heo K, Seo Y, Ahn H, Jung K, Lee $S$, et al. Flattening the curve on COVID-19: South Korea's measures in tackling initial outbreak of coronavirus. Am J Epidemiol 2020:kwaa217.

3. Park Y, Huh IS, Lee J, Kang CR, Cho SI, Ham HJ, et al. Application of testing-tracing-treatment strategy in response to the COVID-19 outbreak in Seoul, Korea. J Korean Med Sci 2020;35(45): e396.

4. Kwon S. Thirty years of national health insurance in South Korea: lessons for achieving universal health care coverage. Health Policy Plan 2009;24(1):63-71.

5. Kim JA, Yoon S, Kim LY, Kim DS. Towards actualizing the value potential of Korea Health Insurance Review and Assessment (HIRA) data as a resource for health research: strengths, limitations, applications, and strategies for optimal use of HIRA data. J Korean Med Sci 2017;32(5):718-728. 
6. Shin HC, Park YT, Lee YT, Jo EC. Healthcare utilization monitoring system in Korea. Healthc Inform Res 2015;21(3):184-190.

7. Kang CR, Lee JY, Park Y, Huh IS, Ham HJ, Han JK, et al. Coronavirus disease exposure and spread from nightclubs, South Korea. Emerg Infect Dis 2020;26(10):2499-2501.
8. Consulate General of the Republic of Korea in Houston. Flattening the curve on COVID-19 - how Korea responded to a pandemic using ICT; 2020 [cited 2020 May 1]. Available from: http://overseas.mofa.go.kr/us-houston-en/brd/m_5573/view. do?seq $=759765$. 